\title{
Frontières
}

\section{Parcours passionnels de la peur et angoisse de mort}

\section{Christiane Kègle}

Volume 12, numéro 2, printemps 2000

Peur bleue...

URI : https://id.erudit.org/iderudit/1074404ar

DOI : https://doi.org/10.7202/1074404ar

Aller au sommaire du numéro

Éditeur(s)

Université du Québec à Montréal

ISSN

1180-3479 (imprimé)

1916-0976 (numérique)

Découvrir la revue

Citer ce document

Kègle, C. (2000). Parcours passionnels de la peur et angoisse de mort.

Frontières, 12(2), 74-76. https://doi.org/10.7202/1074404ar d'utilisation que vous pouvez consulter en ligne.

https://apropos.erudit.org/fr/usagers/politique-dutilisation/ 


\section{Parcours passionnels de la peur et angoisse de mort}

\section{«[...] QUELQUE CHOSE D'EFFROYABLE, UNE SENSATION ATROCE, COMME UNE DÉCOMPOSITION DE L'ÂME, UN SPASME AFFREUX DE LA PENSÉE ET DU COEUR, DONT LE SOUVENIR SEUL DONNE DES FRISSONS.»}

\author{
Christiane Kègle, \\ professeure au Département des litteratures \\ de l'Université Laval.
}

\section{L'ANGOISSE DE MORT EN PARTAGE}

La peur est habituellement décrite comme un phénomène psychologique impliquant une manifestation d'affects face à un danger réel ou appréhendé. Parmi les phénomènes reliés à la peur, l'angoisse de mort est sans doute le plus intense. Durant une période de deuil, ou de deuil pathologique, elle engendre une déstabilisation émotive dont les expressions sont variées: peur du gouffre, terreur schizoïde née d'un sentiment aigu de dépersonnalisation, frayeur provoquée par le retour hallucinant de la personne défunte dans les rêves, cauchemars épouvantables augmentant le rythme cardiaque du rêveur, qui se réveille effrayé et en transes.

Appréhendée dans le contexte néolibéraliste des contraintes budgétaires qui affectent notre système de santé, l'angoisse de mort s'avère le lot du quotidien. On fait grand usage sur la scène publique d'anecdotes entourant la mort, mais est-ce pour mieux en refouler l'empreinte durable dans la psyché? Entourée de non-dit, l'expérience intime de la mort demande à être nommée, circonscrite, apprivoisée par le langage. Aussi, des parcours passionnels de sujets mobilisés par la peur feront l'objet de mon essai. Je ferai appel à un "Je» impliquant une pluralité d'instances discursives: je, écrivant, tu, lisant, il ou elle, désignant les absents et les absentes, vous, qui m'avez fait partager votre douleur. Adoptant une écriture libre, je ferai alterner mes récits avec d'autres voix, celles de l'anthropologue, du sociologue, du philosophe éthicien, de l'essayiste. À la manière des récits polyphoniques de Michel Butor, j'effectuerai des ruptures entre la fiction, inspirée par l'expérience subjective de la mort de l'autre, et un certain reflet du discours social sur la mort.

Dans notre société occidentale, c'est par le truchement $d u$ deuil pathologique, par l'angoisse non socialisée et par la folie que s'exprime le plus clairement la réalité de la conscience muette. (Jean Ziegler ${ }^{1}$ )

Sollicitée par ce funeste et insondable phénomène qui oriente ma vie et scelle ma destinée, je parlerai de ce spectre hideux qui ravît père, mère, conjoint-e, frère, soeur, enfant, amis-es, collègues et jusqu'à notre conscience.

\section{UN DIMANCHE D'AVRIL, AU SÉJOUR DES MORTS}

Je vais me promener dans le cimetière B. Printemps tardif. Petit vent du nord, piquant. Tout en marchant, je lis les noms, les dates, les noms de lieux: seuls signifiants qui n'échappent pas à l'oubli des pierres tombales. Sans m'en rendre compte, je m'enfonce plus avant. Mes pensées vagabondent. J'oublie que je suis dans un cimetière, seule, vulnérable. Arrivée tout au fond, je me heurte à un grand mausolée. Le temps s'est assombri, un nuage gris plane au-dessus de moi, menaçant. Je m'arrête, interdite. Les stèles funéraires m'encerclent; les croix, gris noir, sombres, me dominent de leur hauteur impassible. Un silence sonne l'alerte. Une ombre passe. Je la reconnais. En moi. La peur me donne des ailes aux talons. Affolée, je m'enfuis, craignant d'être retenue vivante dans ce grand caveau dont le temps a grugé l'entrée.

Depuis l'aube des sociétés organisées, les hommes craignent d'être ensevelis vivants, brûlés vifs, déclarés morts, détruits, abandonnés par leurs contemporains, alors qu'ils sont simplement endormis, en état de choc ou drogués. (Jean Ziegler²)

J'atteins bientôt la sortie donnant sur le grand boulevard. Mon coeur se calme pendant que ma cadence ralentit. Mes souvenirs m'emportent vers Aigues-Mortes, puis vers la mer... Tu es quelque part, là-bas, dans ce grand cimetière marin ${ }^{3}$, ô poète! Bercé par les flots où ne passent plus les grands paquebots d'autrefois. Les goélettes, vides, amarrent au Grau-du-Roi. La mer a effacé toute trace de tes pas sur le sable fin.

\section{LE SOLEIL DE JUIN ÉTAIT POURTANT RADIEUX}

Je suis retournée à l'hôpital $P$. de Casablanca. La rotonde est toujours affectée à l'unité des soins intensifs. Un malade vient de subir une transplantation cardiaque.

Je suis engouffrée à nouveau par ce passé qui ne veut pas mourir...

Tu as été ranimé cette nuit. Tu gis, comateux, au milieu des draps défaits. Respiration haletante. Tu transpires, de tous tes pores. Une pompe artificielle. Flux et reflux de ton sang. Ressac tumultueux. Je m'immobilise, interloquée, inquiète. Tu as frôlé la mort; sans moi. Ton corps saura-t-il intégrer cet organe artificiel à son rythme défait? Tu entrouvres les paupières, ne me reconnais pas. Tu parles, tes mots ne font plus 
sens. Phrases désarticulées, bouche pâteuse. Je ne te reconnais plus. Tu ne sais plus qui je suis.

Morphine. Contre ta douleur. Abolit ta conscience. Tu te meurs à toi-même, lentement, inexorablement. Rejeté sur l'autre rive, dans un néant d'être, tu abolis en moi tout espoir. Tu vas mourir, mais je ne veux pas le savoir.

C'est [donc] parce que son savoir sur la mort est extérieur, appris, non inné, que l'homme est toujours surpris par la mort. [...] Aveugle donc naturellement à la mort, l'homme est sans cesse forcé de la réapprendre.

Le traumatisme de la mort est précisément l'irruption

de la mort réelle, de la conscience de la mort, au

coeur de cette cécité. (Edgar Morin ${ }^{4}$ )

L'on m'interdit de rester à ton chevet. Je descends au rezde-chaussée. Passe la nuit, raidie, sur une chaise en bois. Yeux grands ouverts dans la nuit. L'aube me surprend, défaite, anémique. Un garçon distribue aux patients des croissants et du café. Me tend un bol de café au lait. Seule manifestation d'humanité dans ce désert du coeur.

Fébrile. Huit heures au cadran de la vieille horloge. Je m'arrache à mon siège, monte les escaliers, péniblement. Mon regard, furtif, à travers la petite vitre des portes battantes. Tu es là, inconscient. Vivant. Ton coeur bat à un rythme fou. Ma respiration s'emballe.

Impuissante, inutile, expulsée de toute vie. Les cinq minutes sont écoulées, on me demande de sortir. Les jours s'écoulent ainsi, par alternances de visites brèves et de longues heures d'appréhension. Je rentre chez moi le cinquième jour. On me téléphone, sur le coup de minuit..

La chambre du mourant est passée de la maison à l'hô-

pital [...] lieu de la mort solitaire. (Philippe Ariès ${ }^{5}$ )

Le mourant est un gêneur. Puisque la mort elle-même

est occultée, masquée, évacuée et puisque sur elle se

fixe l'ensemble des valeurs négatives de la société, l'ago-

nie ne peut pas avoir de statut autonome. (Jean Ziegler ${ }^{6}$ )

Aphasique, absente, verrouillée. L'expérience du néant est indicible.

Un poulpe m'étreint la nuit, enserre ma gorge, étend ses tentacules sur mon dos, mes bras, mes jambes. Yeux ouverts dans la nuit, je veille.

\section{SEPTEMBRE NOIR}

Plusieurs examens, à la clinique externe. Elle manque de force, n'arrive plus à remonter les escaliers. Médecin en vacances. On consulte un autre collègue du même cabinet. Il examine son dossier. Elle souffre d'anémie sévère, il faut l'hospitaliser ce soir même, avant huit heures. Plus de temps à perdre.

Elle m'attend, désemparée. Son visage, souriant, dissimule mal une appréhension folle. Terreurs secrètes. La rassurer, à tout prix.

Sa jambe, posée sur un pouf, enflée, plus que jamais. Terrorisant.

Quand l'âme se protège par mille subterfuges plus ou moins conscients, le corps, lui, sait.

Il faut partir tout de suite. Elle s'affole, se met à déparler, essaie de plaisanter. Son visage expressif. Son regard intelligent. Elle est pourtant traumatisée.

$\mathrm{Tu}$ as connu des situations analogues: lorsqu'un grand malade sort de chez lui, quelque chose d'imperceptible se passe, un signe, une intuition, un éclair de lucidité. Il sait qu'il ne reviendra plus. Que le quotidien des jours tranquilles vient de s'abolir dans cet instant fulgurant.

Formalités d'usage. Sa chambre. Nos silences, éloquents. Je la quitte vers neuf heures du soir. Fuir l'aveuglante vérité: elle va mourir.
Un matin, sa chambre, peur incontrôlable: plasma sanguin au-dessus d'elle, le sang s'écoule goutte à goutte. Je sors, précipitamment.

Le médecin et moi, en aparté. La vérité. Cancer généralisé. Os. Poumons. Quelques mois à peine. Elle doit quitter l'hôpital. Pas de traitements possibles.

Ça va très vite. Son agonie. Douleurs insupportables. Palpitations. Le médecin est dépassé. Marchette!? En phase terminale!? Codéine!? Non, non, nous vous en supplions, cocktail de Brampton, morphine et autres sédatifs. Vous allez la tuer!

À la maison, soins palliatifs. Ce n'est pas évident. Médication mal administrée (il faut se relayer, les messages circulent mal). Elle entre dans une phase de terreur sans nom, réclame un prêtre.

Sur son visage, le masque de la mort. Ses ongles, trop longs; ses cheveux, hirsutes. Terrorisant.

Durant les derniers instants de l'agonie, le mourant vit

entièrement selon sa perception nouvelle. Aucune

communication n'a plus lieu avec ceux qui l'entou-

rent. La main ne répond plus à la pression amicale.

Le regard du mourant est ailleurs. Une expression

nouvelle et inconnue des vivants apparaît sur son

visage. (Jean Ziegler ${ }^{7}$ )

Des sécrétions. Elle étouffe! Elle étouffe! Pas d'appareil. Pas d'ordonnance: refus du médecin. Ambulance. Coma. Longue attente à l'urgence. Transport de la malade au 5e étage, atmosphère de kermesse (le refoulement de la mort peut prendre plusieurs formes: les infirmières ricanent en balançant la couverture sur laquelle elle est posée).

Nouvelles sécrétions, étouffements. Affolement. Colère. Indignation. Pas d'appareil: il faut attendre l'approbation du médecin (qui est en congé). Sa respiration rauque, pendant deux jours et deux nuits. Au crépuscule, sa tête roule sur l'oreiller, comme une chose molle. F. éclate en sanglots.

On peut refouler la mort. La société marchande nous l'enseigne tous les jours. Dans la vie quotidienne, la mort n'a pas de nom, pas de visage, pas de représentations symboliques.

Dans mon sommeil, un squelette animé, vivant, marche dans ma chambre, se penche sur moi, me sourit, d'une étrange façon. Cet air invitant et sournois m'effraye plus que tout.

Inquiétante étrangeté. Représentation de ma propre mort, la nuit, au-dessus de moi. Invitation à partir avec elle. Ricane devant mon émoi.

Le Soi peut enrober et dissoudre l'idée de la mort, mais à son tour peut être rongé par elle: la conscience obsé-

dante de la mort, en son point extrême, flétrit et pourrit

la vie, et conduit à la folie ou au suicide. (Edgar Morin ${ }^{8}$ )

\section{DÉCEMBRE, SOUS LA NEIGE}

Vous, aussi, avez accompagné l'autre dans la mort. Vous savez ce dont je parle. Malgré les années écoulées, marqués par la fatigue psychique, vos organes dysfonctionnent.

Elle a beaucoup souffert. Cancer généralisé.

Mais ce que vous n'acceptez pas, c'est l'incompréhension des médecins, ce que vous appelez leur abus de pouvoir. Fidèle à vous-même, à votre formation scientifique, à votre façon rigoureuse de faire les choses en tout et pour tout, vous avez veillé des heures et des heures, à son chevet.

Cette agonie-là a aussi été la vôtre.

Vous restez dans sa chambre à elle, luttant avec elle, de toutes vos énergies. Vous prenez soin d'elle, surveillez consciencieusement ses signes vitaux, les notant dans un registre à cette fin, calculant, établissant des corrélations entre tel événement et tel symptôme. Vous avez votre propre théorie sur sa maladie. En vain. On ne vous écoute pas. 
Ultime injure, on profite d'une de vos rares absences pour lui administrer la dose létale.

Cela, vous ne leur pardonnerez jamais. N'avait-elle pas le droit de choisir le moment de partir? N'aviez-vous pas désiré tous les deux, pour elle, une mort naturelle? Une symbiose parfaite, tant que le permettrait son ultime souffle de vie?

Le subconscient du patient est - jusqu'à un certain

stade de l'agonie - habité par un refus profond, abso-

lu, irréductible de sa propre mort. La négation de la

mort par la société marchande constitue bel et bien un

système de la négativité pensée de la mort, mais un

système élémentaire qui ne fait pas sa part à l'homme.

Ce système aliène l'homme [la femme] qui meurt, il le

prive de toute pensée de sa mort, même de toute pen-

sée de négation de sa mort. (Jean Ziegler ${ }^{9}$ )

Depuis son décès, vous vivez une double vie. Le jour, vous retournez à vos équations. La nuit, une vie parallèle fleurit dans votre sombre demeure. Vous établissez des preuves, reconstituez sans fin les événements, construisez des procès imaginaires.

Qui vous entendra?

La solitude appelle la hantise de la mort et la hantise

de la mort referme la solitude. (Edgar Morin ${ }^{10}$ )

\section{UN HIVER TROP LONG}

Elle avait frappé à plusieurs portes, avait consulté plusieurs cabinets de médecins, avant d'être écoutée et entendue. Par un urgentologue. Ses os avaient été détruits. Un médicament, avait-on avancé, en guise d'explication. Mentionné dans la monographie du produit? Plus personne ne fait attention à ça, voyons! Son état postopératoire s'était aggravé. Avait dû quitter sa maison, ses amis, ses proches. Exilée, dans une autre ville, seule avec sa douleur, ses échecs.

J'imagine l'effet désastreux que cela a dû avoir sur elle, fragilisée, démolie, encore si jeune.

Un soir du mois de mars, j'ai ouvert par hasard mon courrier électronique, vers onze heures du soir, avant d'aller au lit. On m'annonçait son décès.

Fulgurant. Incompréhensible.

Elle allait pourtant si bien en décembre...

Le plus effrayant: son silence.

Pourquoi n'a-t-elle pas appelé au secours? Pourquoi n'a-telle pas manifesté des signes de détresse?

Elle est partie.

Un choix, dit-on.

Surdose de médicaments pour lutter contre la douleur.

Toute névrose est une tentative régressive de réconci-

liation avec le milieu. Le suicide, rupture suprême, est la réconciliation suprême, désespérée, avec le monde.

(Edgar Morin ${ }^{11}$ )

\section{LES QUATRE SAISONS}

Une nouvelle à la radio: un jeune garçon est mort, ayant reçu par perfusion une trop grande quantité de liquide. À l'hôpital, trop affairé, on avait oublié de contrôler le débit, après les antibiotiques.

Une amie me raconte: «Ma fille avait très mal au ventre; on a consulté plusieurs fois. On ne savait pas ce qu'elle avait. On a fait plusieurs diagnostics, contradictoires. On est même allé jusqu'à lui recommander de la physiothérapie, de la relaxation... Finalement, elle a été opérée d'urgence. Appendicite aiguë. Hospitalisation brève, virage ambulatoire. Sa plaie s'est réouverte. Infection. On est retourné à l'urgence, deux ou trois fois. A failli mourir. Mon dieu!»

La société marchande a occulté la mort. Nos politiciens la dénient sur la place publique. Avec une attitude de pure désin- volture. Le visage couvert d'un masque souriant, sournois. Ils font les comptes, établissent des quotas, restreignent les médicaments, les appareils, les examens. Choisissent qui mourra, qui vivra.

Pour nous rassurer, ils [les politiciens] nous mentent, et nous traitent ensuite comme des imbéciles en insistant sur des choses que nous savons tous être fausses. [...] Il est extrêmement destructeur de permettre à l'efficience de diriger la machine, alors que les idées clopinent derrière. Et c'est un symptôme classique d'un système qui a perdu les pédales. (John R. Saul ${ }^{12}$ )

Il y a là un problème d'éthique sociale que les pouvoirs en place refusent habituellement de prendre en compte [...] Prendre une responsabilité sans devoir jamais en répondre, n'est-ce pas là le plus complet arbitraire? Ces choix sont arbitraires au sens où ils ne sont pas concertés avec l'ensemble des personnes concernées. Une machine à déshumaniser la plupart des humains s'est mise en place au profit d'une minorité [...] Ces choix arbitraires sont délétères, voire mortifères. (Jean-François Malherbe ${ }^{13}$ )

Je suis revenue sur mes pas. Au cimetière B. Ai rencontré des personnes qu'on dit âgées, bien vivantes, sereines, souriantes dans le soleil matinal. M'ont amenée voir une belle sculpture, toute blanche, en haut d'une stèle, sur un fond de bourgeons vert tendre, prêts à éclore. Une belle dame, allégorie d'un printemps éternel, sourit, épanchée sur ses morts, qu'elle protège. Son visage, le drapé de sa tunique, les torsades de ses cheveux, ses mains répandant des roses de pierre. On admire cette belle réussite sur le plan esthétique. Pacifiant.

Un peu plus loin, une large et haute clôture en bois. Derrière: un charnier. Des pierres tombales cassées, mutilées, empilées par la machinerie lourde. Le dépotoir de la mort. Scandaleux! Le patrimoine culturel, aboli, la mémoire des morts, bafouée. L'éternité équivaut à vingt-cinq ans dans les contrats funèbres qui lient les citoyens aux fabriques des paroisses.

Les civilisations sont mortelles. L'humanité est promise à la mort. La terre mourra. Et les mondes et les soleils. Et l'univers lui-même, gigantesque explosion lente. La mort humaine, déjà vide infini, se dilate sur tous les plans du cosmos, de plus en plus vide et infinie: elle est comme l'univers, en expansion. Tout renvoie donc l'individu solitaire à une solitude de plus en plus misérable au creux d'un néant sans limites. (Edgar Morin ${ }^{14}$ )

\section{NOTES}

1 Jean ZIEGLER, Les Vivants et la mort, Paris, Seuil, Collection Points, 1975, p. 41.

2 Ibid., p. 82.

3 Allusion au poème célèbre de Paul Valéry.

4 Edgar MORIN, L'Homme et la mort, Paris, Seuil, Collection Points, 1970, p. 72 et 73.

5 Philippe ARIES, L'Homme devant la mort, Paris, Seuil, tome 2, Collection Points-Histoire, 1977, p. 280.

6 ZIEGLER, ibid. note 1, p. 139.

7 Ibid., p. 162.

8 MORIN, ibid. note 4 , p. 75

9 ZIEGLER, ibid. note 1, p. 79.

10 MORIN, ibid. note 4, p. 304.

11 Ibid., p. 59.

12 "La santé et les soins à la fin du XXe siècle: des symptômes inquiétants", dans Ça urge! Le système de santé canadien a-t-il un avenir?, sous la direction de Margaret A. SOMERVILLE. Montréal, Fidès, 1999, p. 6 et 11 .

13 Jean-François MALHERBE, L'éthique clinique en situation de pénurie. Un questionnement, Montréal, Fidès, CHS, 1997, p. 37.

14 MORIN, ibid. note 4 , p. 305. 